\title{
A seed dresser's perspective of seed production
}

\section{PETER McCAW}

Farmer, Ashburton

\section{Objective}

The objective is to optimise financial return to the seed grower by saving the maximum amount of seed at the highest possible quality standards.

It is possible to always achieve the highest grades by dressing* a line very hard and discarding a large proportion of the sample, with a loss of potential value to the grower. It is also possible to take out too little of the empty seed and weeds, with a consequential loss of value to the grower which may equal that of the first instance. The trick is'to achieve a balance wherein all possible good seed is saved while the required standards for that type of seed are still met.

It is not possible to clean out rubbish and weeds without also taking off a fraction of good seed. The size of that fraction depends on how much foreign matter is there to start with - and that depends on how well the grower controlled weeds in his crop and on his ability to set up his combine to produce a good sample at harvest time. Weight losses from the farmer's line of seed brought in for cleaning can range from $5 \%$ to $50 \%$. Much of this variation can be put down to grower skills - or lack of. A very large part of the total cleaning job should be done in the paddock, as the seed dresser cannot make a silk purse from a sow's ear.

Over recent years, seed production_ has become more and more the job of specialist growers. The fine margins of profit have gradually removed the catchcrop grower from the scene. In the past, it was common for a mixed sheep and cropping farmer to plant a stand of grass and clover with the idea that if his sheep didn't need it, a seed crop could be taken. This often meant the stand was grazed through lambing, thereby removing the main seed-head tillers and resulting in a small yield of light seed. Successful management for high yield and quality begins with the selecting of a paddock in which to grow the crop and thereafter never losing sight of the fact that this is a specialist crop with yield and quality taking precedence over all else.

\section{Storage and handling}

The industry has been quick to adapt to bulk handling methods for moving seed from the combine to the seed dresser. Very little seed is delivered in sacks, and that

"dressing" covers all operations to seed from harvest
onwards.

which is would usually be tipped into bulk boxes for subsequent handling. Providing the seed is sufficiently dry, it can be safely stored in silos or pallet-based bulk boxes. Out-of-condition seed with too high a moisture content can heat up in storage, causing fungal activity which, in turn, generates more heat. Damage can range from a small drop in germination and vigour through to spontaneous combustion. The fungus involved is Aspergillis glaucus which, given the right conditions, can destroy a year's effort in a couple of days. The amount of damage is determined by the ratio of heat to time. High temperatures for a short time or medium temperatures over a long time can do the damage. Seed naturally experiences high temperatures in the field on a hot day, but because this is always followed by cool nights, no damage is done to the seed.

It is very important to keep each line of seed properly identified throughout the dressing procedure and this is achieved by labelling each container with stick-on labels supplied by MAF. These show the seedline reference number as well as area of production and grade of seed. These details follow the seed through all stages from field inspection to the consumer. (The sack of seed the end user buys has these same details on an attached tag.) A seed-line can therefore be traced back to the seed used to produce it. The certification system ensures that seed in any given sack 'is produced in the area specified and meets certain minimum purity standards. While these standards are high, it is still a good idea for a farmer purchasing seed to make sure he sights a copy of the purity and germination certificate for that line, as the system does not cover germination. It is important to be satisfied of quality in all respects.

\section{Dressing or cleaning}

The principles of cleaning seed have changed little over the years, but there have been major gains from improved machinery design.

The science of seed cleaning revolves around the differences in seeds. These sometimes subtle differences are:

1. Heavy or light: Light seed can be lifted off by air suction.

2. Individual seed size: A sieve of the correct size and hole shape can be used to obtain separation.

3. Seed shape versus weed shape:

(a) Fat or thin: separation by sieving or by a jumping sieve to pass the thin seed through a smaller hole or slot on end or on its edge. 
(b) Long or short: separation using either an indent cylinder or disc separator or, in some cases, a suitable sieve.

(c) Round or angular: a triangular-hole sieve will allow a dock seed to drop through but not a roundbodied grain or seed. A round seed will roll, whereas a flat-sided or angular seed will slide more slowly on an incline. This difference is used to obtain separation with a spiral separator where the seed is poured down a spiral a bit like a large auger flighting. The round seed rolls faster and therefore centrifugal force takes it out over the edge into a separate collection tray. This machine is commonly used to separate white clover from lotus but is of limited capacity.

4. Texture: A rough-coated or cracked seed will stick to a rotating velvet covered roller and be carried away from smooth seeds which are left behind. Another option is to use a magnetic separator in which the seed is moistened and mixed with fine iron powder. As the seed dries, the smooth coated seeds lose their powder covering, whereas the cracked or rough seeds hold enough iron to be attracted and retained by a magnet. 5. Bounce: Some seeds bounce well. This ability can be used to separate peas from clods of earth of similar size and weight which have managed to pass through the system. Dropped on an inclined sheet of glass, the peas bounce clear while the earth clots and other matter slide off.

6. Specific gravity: A specific gravity separator fluidifies the seed layer of supporting the seed on an air flow from beneath. With the tray or table of the machine on an incline and 'reciprocating', the heavier seed sinks to the table surface and is carried to the high end, while the light seed works its way to the top of the layer and floats downhill to be collected in different grades from points lower down the table. This machine can be used on a wide range of seeds.

7. Electricity: Variations in a seed's ability to take and hold an electrical charge can be used in a somewhat similar manner to a magnetic separator. The positively charged seeds are attracted to a negatively charged roller and therefore hold on longer than uncharged seeds.

8. Colour: Electronic colour sorters examine a single stream of seeds dropped passed sensors which are able to detect subtle differences in shade. When an unwanted seed is detected, it is blown sideways from the rest by a short sharp blast of compressed air. This expensive and slow system is used only on specialty lines of seed.

\section{Plant variety rights}

In recent years more and more seed varieties traded have come under the Plant Variety Rights scheme (PVR). The purpose of this scheme is to provide the variety's breeder with a return through a royalty on seed traded. The control necessary to achieve this is provided for in law which enables a seed breeder to determine the area grown for seed production and who shall market the resultant crop. This proprietary ownership of varieties means a breeder can put the effort into developing a new variety, secure in the knowledge that he will not lose control over seed production when it enters the market place, and that his efforts will be financially rewarded. A number of excellent free varieties are still around which were in circulation before the advent of PVR, and some of these will be around for many years to come, notably 'Grasslands Nui' ryegrass and 'Grasslands Huia' white clover, which still dominate the market.

\section{Overseas multiplication}

In the last 2 or 3 years a substantial trade has developed between New Zealand and both the U.S and European countries, wherein an overseas company contracts growers to produce seed of their variety for re-export and sale in the country of origin. Many such varieties are specialist types, bred for turf production rather than forage use, but clovers are subsequently sold in direct competition to our New Zealand varieties. New Zealand produces the lion's share of all white clover traded internationally, with 'Grasslands Huia' still making up the bulk of it. Our climate is eminently suited to white clover production and we do the job better than anyone else in the world. Our advantage with turf grass production lies in the fact that our seasons are 6 months different from those of the Northern Hemisphere. This staggered production is of great value to seed companies, both in reduced inventories required and as a hedge against climatic disaster in any one production area.

The U.S. turf industry standards for purity are very high and not easy for growers to achieve. Growers are putting time and effort into getting their fields totally clean of other grasses and weeds, and in return are obtaining good premiums for top grade seed. A few seeds of a different grass left in a line of forage grass is generally of minor importance, but for fine turf it can mean the difference between a sale or no sale. We can be proud that we have the expertise and infrastructure to attract this new and specialised business to New Zealand. The opportunity has come at a good time for growers, coinciding as it did with a substantial downturn in demand for our traditional varieties. Many specialist growers are now committing most of their area to production of overseas varieties, and this should create healthy competition for the industry here.

\section{Summary}

We have an industry which can foot it with the rest of the world. Our growers have the expertise. Our seed processors can produce the quality demanded and our seed certification and testing schemes ensure that quality.

An important part of the grasslands industry, seed cleaners look forward with confidence to moving into the future, hand in hand with the rest of the grasslands team 